Publ. Math. Debrecen

97/1-2 (2020), 77-84

DOI: $10.5486 /$ PMD.2020.8699

\title{
Perfect numerical semigroups with embedding dimension three
}

\author{
By MARÍA ÁNGELES MORENO-FRÍAS (Puerto Real) \\ and JOSÉ CARLOS ROSALES (Granada)
}

\begin{abstract}
A numerical semigroup is perfect if it has no isolated gaps. In this paper, we will characterize the perfect numerical semigroups with embedding dimension three, and we show how to obtain them all. Also, we obtain formulas for each of the genus and the pseudo-Frobenius numbers of these semigroups.
\end{abstract}

\section{Introduction}

We denote by $\mathbb{Z}$ and $\mathbb{N}$ the set of integers and the nonnegative integers numbers, respectively.

A submonoid of $(\mathbb{N},+)$ is a subset $M$ of $\mathbb{N}$ which is closed by the sum and $0 \in M$. A numerical semigroup is a submonoid $S$ of $(\mathbb{N},+)$ such that $\mathbb{N} \backslash S=\{x \in$ $\mathbb{N} \mid x \notin S\}$ is finite.

If $A$ is a non-empty subset of $\mathbb{N}$, we denote by $\langle A\rangle$ the submonoid of $(\mathbb{N},+)$ generated by $A$, that is, $\langle A\rangle=\left\{\lambda_{1} a_{1}+\cdots+\lambda_{n} a_{n} \mid n \in \mathbb{N} \backslash\{0\},\left\{a_{1}, \ldots, a_{n}\right\} \subseteq A\right.$ and $\left.\left\{\lambda_{1}, \ldots, \lambda_{n}\right\} \subseteq \mathbb{N}\right\}$. It is well known (for example, see [10, Lemma 2.1]) that $\langle A\rangle$ is a numerical semigroup if and only if $\operatorname{gcd}(A)=1$.

If $M$ is a submonoid of $(\mathbb{N},+)$ and $M=\langle A\rangle$, then we say that $A$ is a system of generators of $M$. Moreover, if $M \neq\langle B\rangle$ for every $B \subsetneq A$, then we say that $A$ is a minimal system of generators of $M$. In [10, Corollary 2.8] it is shown that every submonoid of $(\mathbb{N},+)$ has a unique minimal system of generators, which

Mathematics Subject Classification: Primary: 20M14; Secondary: 11D07.

Key words and phrases: embedding dimension, Frobenius number, genus, numerical semigroup, perfect numerical semigroup, pseudo-Frobenius numbers.

This paper is supported by MTM2017-84890-P and by Junta de Andalucía groups FQM-298 and FQM-343. 
in addition is finite. We denote by $\operatorname{msg}(M)$ the minimal system of generators of $M$. The cardinality of $\operatorname{msg}(M)$ is called the embedding dimension of $M$ and will be denoted by e $(M)$. It is clear that $\mathbb{N}$ is the unique numerical semigroup with embedding dimension one.

If $S$ is a numerical semigroup, the elements of $\mathbb{N} \backslash S$ are known as the gaps of $S$. We will say that a gap $h$ of $S$ is isolated if $\{h-1, h+1\} \subseteq S$. A perfect numerical semigroup is a numerical semigroup without isolated gaps. $\mathbb{N}$ is a perfect numerical semigroup.

The perfect numerical semigroups were introduced in [3]. They are a family of numerical semigroups, whose name comes from topology, specifically the concept of a perfect set (set without isolated points). The family of perfect semigroups is arranged in a tree, and this construction allows us to study certain aspects and properties of them (see [3]).

The importance of the study of perfect numerical semigroups lies in the fact that, so far, in the tree of the families of numerical semigroups studied (see [7], [5] and [6]), children had one more gap than their parents; however, in the family of perfect numerical semigroups children have now two gaps more than their parents do.

Let $M$ be a submonoid of $(\mathbb{N},+)$ such that $M \neq\{0\}$. The multiplicity of $M$, denoted by $\mathrm{m}(M)$, is the smallest positive integer which belongs to $M$. From [10, Propositions 2.2 and 2.10], we deduce that $\mathrm{e}(M) \leq \mathrm{m}(M)$. We will say that a numerical semigroup has maximal embedding dimension if $\mathrm{e}(S)=\mathrm{m}(S)$.

If $S$ is a numerical semigroup, then the greatest integer number that does not belong to $S$ is called the Frobenius number of $S$, and it will be denoted by $\mathrm{F}(S)$. Note that $\mathrm{F}(\mathbb{N})=-1$ and $\mathrm{F}(S) \in \mathbb{N} \backslash\{0\}$ if $S \neq \mathbb{N}$.

The main aim of the study of perfect numerical semigroups is to advance in the resolution of important open problems existing in the field of numerical semigroups such as finding a formula for the Frobenius number (see [4]) in embedding dimension greater or equal than 3 and WILF's conjecture (see [11]), among others.

In [3], some properties of the perfect numerical semigroups have already been studied: construction of an algorithmic procedure that allows us to obtain all the perfect semigroups with a fixed multiplicity, perfect numerical semigroups with maximal embedding dimension, the perfect closure of a numerical semigroup, etc.

In this work, we will prove that numerical semigroups with embedding dimension two are not perfect (see Corollary 4), and we will study perfect numerical semigroups with embedding dimension three, in order to study the behaviour of these semigroups and to see if that can be generalized to semigroups with greater embedding dimension. In Proposition 8, we prove that a numerical semigroup $S$ 
with embedding dimension three is perfect if and only if $\mathrm{F}(S)-1 \notin S$. The main result of Section 3 is Theorem 17, which explicitly presents how all the perfect semigroups with embedding dimension three are generated.

\section{Pseudo-Frobenius numbers}

Let $S$ be a numerical semigroup. Following the notation introduced in [8], we say that an element $x \in \mathbb{Z} \backslash S$ is a pseudo-Frobenius number of $S$ if $x+s \in S$ for all $s \in S \backslash\{0\}$. We will denote by $\operatorname{PF}(S)$ the set of pseudo-Frobenius numbers of $S$, and its cardinality will be called the type of $S$, denoted by $\mathrm{t}(S)$. From the definition it easily follows that $\mathrm{F}(S) \in \mathrm{PF}(S)$, in fact, it is the maximum of this set.

Lemma 1. If $S$ is a perfect numerical semigroup, then $\mathrm{F}(S)-1 \notin S$.

Proof. If $S=\mathbb{N}$, then $\mathrm{F}(S)-1=-2 \notin S$. If $S \neq \mathbb{N}$, then $\mathrm{F}(S) \in \mathbb{N} \backslash S$ and $\mathrm{F}(S)+1 \in S$. As $S$ is perfect, we have that $\mathrm{F}(S)$ is not an isolated gap. Therefore $\mathrm{F}(S)-1 \notin S$.

The following result appears in [8, Proposition 12].

Lemma 2. Let $S$ be a numerical semigroup and $x \in \mathbb{Z}$. Then $x \notin S$ if and only if there exists $f \in \operatorname{PF}(S)$ such that $f-x \in S$.

Let $S$ be a numerical semigroup with $\mathrm{t}(S)=1$. Then $\operatorname{PF}(S)=\{\mathrm{F}(S)\}$. If $S \neq \mathbb{N}$, then $1 \notin S$, and applying Lemma 2 , we have $\mathrm{F}(S)-1 \in S$. Hence, according to Lemma $1, S$ is not a perfect numerical semigroup. Therefore, we have the following result.

Proposition 3. If $S$ is a numerical semigroup such that $S \neq \mathbb{N}$ and $\mathrm{t}(S)=1$, then $S$ is not a perfect numerical semigroup.

In [10, Example 2.22] it is proven that if $S$ is a numerical semigroup with $\mathrm{e}(S)=2$, then $\mathrm{t}(S)=1$. Thus, applying Proposition 3 , we have the following result.

Corollary 4. If $S$ is a numerical semigroup and e $(S)=2$, then $S$ is not a perfect numerical semigroup.

Lemma 5. Let $S$ be a numerical semigroup such that $\mathrm{t}(S)=2$. Then $\mathrm{PF}(S)=\{\mathrm{F}(S)-1, \mathrm{~F}(S)\}$ if and only if $\mathrm{F}(S)-1 \notin S$. 
Proof. If $\mathrm{PF}(S)=\{\mathrm{F}(S)-1, \mathrm{~F}(S)\}$, then $\mathrm{F}(S)-1 \in \mathbb{Z} \backslash S$, and therefore, $\mathrm{F}(S)-1 \notin S$.

Conversely, t $(S)=2$, implies $S \neq \mathbb{N}$. If $\mathrm{F}(S)-1 \notin S$, then $\mathrm{F}(S)-1 \in \mathbb{Z} \backslash S$. Now $S \neq \mathbb{N}$, implies $1 \notin S$. Consequently, if $s \in S \backslash\{0\}$, we have $\mathrm{F}(S)-1+s \in S$, because $\mathrm{F}(S)-1+s>\mathrm{F}(S)$. Hence $\mathrm{F}(S)-1 \in \mathrm{PF}(S)$. As $\mathrm{t}(S)=2$, we have $\mathrm{PF}(S)=\{\mathrm{F}(S)-1, \mathrm{~F}(S)\}$, since $\mathrm{F}(S) \in \mathrm{PF}(S)$.

Theorem 6. Let $S$ be a numerical semigroup with $\mathrm{t}(S)=2$. Then, $S$ is a perfect numerical semigroup if and only if $\mathrm{F}(S)-1 \notin S$.

Proof. If $S$ is a perfect numerical semigroup, then by Lemma 1, we know that $\mathrm{F}(S)-1 \notin S$. Conversely, if $\mathrm{F}(S)-1 \notin S$, then by Lemma 5 , we have $\mathrm{PF}(S)=\{\mathrm{F}(S)-1, \mathrm{~F}(S)\}$. Now, we suppose that $h$ is a gap in $S$. That is, $h \in \mathbb{N} \backslash S$. Then, by Lemma 2 , we have $\mathrm{F}(S)-h \in S$ or $\mathrm{F}(S)-1-h \in S$. We will study these two cases. If $\mathrm{F}(S)-h \in S$, as $\mathrm{F}(S)-1 \notin S$, we deduce that $\mathrm{F}(S)-1-(\mathrm{F}(S)-h) \notin S$, and therefore $h-1 \notin S$. If $\mathrm{F}(S)-1-h \in S$, as $\mathrm{F}(S) \notin S$, we deduce that $\mathrm{F}(S)-(\mathrm{F}(S)-1-h) \notin S$, and therefore $h+1 \notin S$. This way, we have proven that given a gap $h$ in $S$, then also either $h-1$ or $h+1$ is a gap in $S$. Hence, $S$ is a perfect numerical semigroup.

The following result is deduced from [2, Theorem 11].

Lemma 7. If $S$ is a numerical semigroup with $\mathrm{e}(S)=3$, then $\mathrm{t}(S) \in\{1,2\}$.

Proposition 8. Let $S$ be a numerical semigroup with $e(S)=3$. Then $S$ is a perfect numerical semigroup if and only if $\mathrm{F}(S)-1 \notin S$.

Proof. By Lemma 1, we know that if $S$ is a perfect numerical semigroup, then $\mathrm{F}(S)-1 \notin S$. Conversely, if $\mathrm{F}(S)-1 \notin S$, then we deduce that $\mathrm{F}(S)-1 \in$ $\mathrm{PF}(S)$. Applying Lemma 7 , we have $\mathrm{PF}(S)=\{\mathrm{F}(S)-1, \mathrm{~F}(S)\}$. Therefore, $\mathrm{t}(S)=2$, and applying Theorem 6 , we have that $S$ is a perfect numerical semigroup.

We finish this section showing an example which proves that Proposition 8 is not true for $\mathrm{e}(S)=4$.

Example 9. Let $S=\{0,5,7,10, \rightarrow\}=\langle 5,7,11,13\rangle$ (the symbol $\rightarrow$ means that every integer greater than 10 belongs to the set). Then $\mathrm{e}(S)=4, \mathrm{~F}(S)=9$ and $\mathrm{F}(S)-1=8 \notin S$. The numerical semigroup $S$ is not perfect, since 6 is an isolated gap of $S$. 


\section{The six parameters}

Let $S$ be a numerical semigroup and $n \in S \backslash\{0\}$, the Apéry set of $n$ in $S$ (named so in honour of [1]) is $\operatorname{Ap}(S, n)=\{s \in S \mid s-n \notin S\}$. The following result appears in [10, Lemma 2.4].

Lemma 10. If $S$ is a numerical semigroup and $n \in S \backslash\{0\}$, then $\operatorname{Ap}(S, n)=$ $\{0=w(0), w(1), \ldots, w(n-1)\}$, where $w(i)$ is the least element of $S$ congruent with $i$ modulo $n$ for all $i \in\{0, \ldots, n-1\}$.

Notice that as a consequence of the previous lemma, the cardinality of $\operatorname{Ap}(S, n)$ is $n$.

If $S$ is a numerical semigroup, then we can define on $\mathbb{Z}$ the following order relation: $a \leq_{S} b$ if $b-a \in S$. The following result is deduced from [2, Proposition $8]$.

Proposition 11. If $S$ is a numerical semigroup, $n \in S \backslash\{0\},\left\{w_{1}, \ldots, w_{t}\right\}=$ Maximals $_{\leq_{S}}(\operatorname{Ap}(S, n))$, then $\operatorname{PF}(S)=\left\{w_{1}-n, \ldots, w_{t}-n\right\}$.

The following result has an immediate proof.

Lemma 12. Let $S$ be a numerical semigroup and $\operatorname{msg}(S)=\left\{n_{1}, n_{2}, \ldots, n_{e}\right\}$, then $\operatorname{Ap}\left(S, n_{1}\right) \subseteq\left\langle n_{2}, \ldots, n_{e}\right\rangle$.

Proposition 13. Let $S$ be a numerical semigroup such that $\mathrm{F}(S)-1 \notin S$. If $\operatorname{msg}(S)=\left\{n_{1}, \ldots, n_{e}\right\}$ and $\mathrm{e}(S) \geq 3$, then $\operatorname{gcd}\left\{n_{2}, \ldots, n_{e}\right\}=1$.

Proof. It is clear that $\{\mathrm{F}(S), \mathrm{F}(S)-1\} \subseteq \mathrm{PF}(S)$. Therefore, we have $\left\{\mathrm{F}(S)+n_{1}, \mathrm{~F}(S)-1+n_{1}\right\} \subseteq \operatorname{Ap}\left(S, n_{1}\right)$. Then, by Lemma 12 , we have $\left\{\mathrm{F}(S)+n_{1}, \mathrm{~F}(S)-1+n_{1}\right\} \subseteq\left\langle n_{2}, \ldots, n_{e}\right\rangle$. As $\mathrm{F}(S)+n_{1}, \mathrm{~F}(S)-1+n_{1}$ are two consecutive integers, we get $\operatorname{gcd}\left\{\mathrm{F}(S)+n_{1}, \mathrm{~F}(S)-1+n_{1}\right\}=1$, and hence $\operatorname{gcd}\left\{n_{2}, \ldots, n_{e}\right\}=1$.

Note that in the previous proposition, the system of generators is not ordered. Therefore, if $S$ is a perfect numerical semigroup, by Lemma 1, we can assert that $\mathrm{F}(S)-1 \notin S$, and from Proposition 13, we have the following result.

Corollary 14. Let $S$ be a perfect numerical semigroup and $\operatorname{msg}(S)=$ $\left\{n_{1}, n_{2}, n_{3}\right\}$. Then $n_{1}, n_{2}$ and $n_{3}$ are pairwise relatively prime positive integers.

From [9, Theorem 9, Corollary 14], we deduce the following proposition. It shows how we can build all numerical semigroups with embedding dimension three such that their minimal generators are pairwise relatively prime. Moreover, we also see what their pseudo-Frobenius numbers are. 
Proposition 15. If $a_{12}, a_{13}, a_{21}, a_{23}, a_{31}$ and $a_{32}$ are positive integers such that $m_{1}=a_{12} a_{13}+a_{12} a_{23}+a_{13} a_{32}, m_{2}=a_{13} a_{21}+a_{21} a_{23}+a_{23} a_{31}$ and $m_{3}=$ $a_{12} a_{31}+a_{21} a_{32}+a_{31} a_{32}$ are pairwise relatively prime, then $\left\langle m_{1}, m_{2}, m_{3}\right\rangle$ is a numerical semigroup with embedding dimension three. Conversely, every numerical semigroup with embedding dimension three and with a minimal system of generators pairwise relatively prime has this form. Additionally, $\mathrm{PF}\left(\left\langle m_{1}, m_{2}, m_{3}\right\rangle\right)=$ $\left\{f_{1}=-a_{12} a_{13}-a_{12} a_{23}-a_{12} a_{31}-a_{13} a_{21}-a_{13} a_{32}-a_{21} a_{23}-a_{21} a_{32}-a_{23} a_{31}-\right.$ $a_{31} a_{32}+a_{12} a_{13} a_{21}+a_{12} a_{21} a_{23}+a_{12} a_{13} a_{31}+2 a_{12} a_{23} a_{31}+a_{13} a_{21} a_{32}+a_{21} a_{23} a_{32}+$ $\left.a_{13} a_{31} a_{32}+a_{23} a_{31} a_{32}, f_{2}=f_{1}+a_{13} a_{21} a_{32}-a_{12} a_{23} a_{31}\right\}$.

Remark 16. Note that if $m_{1}, m_{2}$ and $m_{3}$ are the positive integers from Proposition 15 , then $\mathrm{F}\left(\left\langle m_{1}, m_{2}, m_{3}\right\rangle\right)=\max \left\{f_{1}, f_{2}\right\}$ and $\mathrm{PF}\left(\left\langle m_{1}, m_{2}, m_{3}\right\rangle\right)=\left\{\mathrm{F}\left(\left\langle m_{1}\right.\right.\right.$, $\left.\left.\left.m_{2}, m_{3}\right\rangle\right), \mathrm{F}\left(\left\langle m_{1}, m_{2}, m_{3}\right\rangle\right)-\left|a_{13} a_{21} a_{32}-a_{12} a_{23} a_{31}\right|\right\}$, where $|z|$ denotes the absolute value of $z$.

Theorem 17. If $a_{12}, a_{13}, a_{21}, a_{23}, a_{31}$ and $a_{32}$ are positive integers such that $m_{1}=a_{12} a_{13}+a_{12} a_{23}+a_{13} a_{32}, m_{2}=a_{13} a_{21}+a_{21} a_{23}+a_{23} a_{31}$ and $m_{3}=a_{12} a_{31}+$ $a_{21} a_{32}+a_{31} a_{32}$ are pairwise relatively prime, and $\left|a_{23} a_{12} a_{31}-a_{32} a_{13} a_{21}\right|=1$, then $\left\langle m_{1}, m_{2}, m_{3}\right\rangle$ is a perfect numerical semigroup with embedding dimension three. Moreover, every perfect numerical semigroup with embedding dimension three has this form.

Proof. From Proposition 15 and Remark 16, we easily deduce that $S=$ $\left\langle m_{1}, m_{2}, m_{3}\right\rangle$ is a numerical semigroup with embedding dimension three and $\mathrm{PF}(S)=\{\mathrm{F}(S), \mathrm{F}(S)-1\}$. Then, $\mathrm{F}(S)-1 \notin S$, and applying Proposition 8 , we have that $S$ is a perfect numerical semigroup.

Let $T$ be a perfect numerical semigroup with embedding dimension three. From Corollary 14, we know that if $\operatorname{msg}(T)=\left\{n_{1}, n_{2}, n_{3}\right\}$, then $n_{1}, n_{2}$ and $n_{3}$ are pairwise relatively prime positive integers. Applying now Proposition 15, we deduce that there exist positive integers $a_{12}, a_{13}, a_{21}, a_{23}, a_{31}$ and $a_{32}$ such that $n_{1}=a_{12} a_{13}+a_{12} a_{23}+a_{13} a_{32}, n_{2}=a_{13} a_{21}+a_{21} a_{23}+a_{23} a_{31}$ and $n_{3}=a_{12} a_{31}+$ $a_{21} a_{32}+a_{31} a_{32}$. As $T$ is perfect, by Lemma 1 we know that $\mathrm{F}(T)-1 \notin T$. Using now the Lemmas 5 and 7 , we obtain $\operatorname{PF}(T)=\{\mathrm{F}(T), \mathrm{F}(T)-1\}$. By Remark 16, we can assert $\left|a_{23} a_{12} a_{31}-a_{32} a_{13} a_{21}\right|=1$.

If $S$ is a numerical semigroup, then the cardinality of $\mathbb{N} \backslash S$ is called the genus of $S$, and we denote it by $\mathrm{g}(S)$. The following result is deduced from $[9$, Corollary 18].

Proposition 18. If $a_{12}, a_{13}, a_{21}, a_{23}, a_{31}$ and $a_{32}$ are positive integers such that $m_{1}=a_{12} a_{13}+a_{12} a_{23}+a_{13} a_{32}, m_{2}=a_{13} a_{21}+a_{21} a_{23}+a_{23} a_{31}$ and $m_{3}=$ 
$a_{12} a_{31}+a_{21} a_{32}+a_{31} a_{32}$ are pairwise relatively prime, then $\mathrm{g}\left(\left\langle m_{1}, m_{2}, m_{3}\right\rangle\right)=$ $\frac{1}{2}\left(1-a_{12} a_{13}-a_{12} a_{23}-a_{12} a_{31}-a_{13} a_{21}-a_{13} a_{32}-a_{21} a_{23}-a_{21} a_{32}-a_{23} a_{31}-\right.$ $a_{31} a_{32}+a_{12} a_{13} a_{21}+a_{12} a_{21} a_{23}+a_{12} a_{13} a_{31}+2 a_{12} a_{23} a_{31}+2 a_{13} a_{21} a_{32}+a_{13} a_{31} a_{32}+$ $\left.a_{21} a_{23} a_{32}+a_{23} a_{31} a_{32}\right)$.

We finish this work illustrating the previous results with an example.

Example 19. Let $a_{12}=2, a_{13}=5, a_{21}=1, a_{23}=7, a_{31}=3$ and $a_{32}=4$. Then, $m_{1}=1 \cdot 5+1 \cdot 7+5 \cdot 4=32, m_{2}=5 \cdot 1+1 \cdot 7+7 \cdot 3=33$ and $m_{3}=$ $1 \cdot 3+1 \cdot 4+3 \cdot 4=19$ are pairwise relatively prime, and $\left|a_{23} a_{12} a_{31}-a_{32} a_{13} a_{21}\right|=$ $|7 \cdot 1 \cdot 3-4 \cdot 5 \cdot 1|=|21-20|=1$. Applying Theorem 17 , we can assert that $S=\langle 19,32,33\rangle$ is a perfect numerical semigroup with embedding dimension three. By Proposition 15, we know that $\operatorname{PF}(S)=\{177,176\}$. So, $\mathrm{F}(S)=177$. Now, applying Proposition 18, we have $\mathrm{g}(S)=99$.

ACKNOwledGements. The authors would like to thank the referees for their useful comments and suggestions that helped to improve this work.

\section{References}

[1] R. ApÉry, Sur les branches superlinéaires des courbes algébriques, C. R. Acad. Sci. Paris 222 (1946), 1198-1200.

[2] R. Fröberg, C. Gottlieb and R. Häggkvist, On numerical semigroups, Semigroup Forum 35 (1987), 63-83.

[3] M. A. Moreno-Frías and J. C. Rosales, Perfect numerical semigroups, Turkish J. Math. 43 (2019), 1742-1754.

[4] J. L. Ramírez Alfonsín, The Diophantine Frobenius problem, Oxford University Press, Oxford, 2005.

[5] A. M. Robles-Pérez and J. C. Rosales, Frobenius pseudo-varieties in numerical semigroups, Ann. Mat. Pura Appl. (4) 194 (2015), 275-287.

[6] A. M. Robles-PÉrez and J. C. Rosales, Frobenius restricted varieties in numerical semigroups, Semigroup Forum 97 (2018), 478-492.

[7] J. C. Rosales, Families of numerical semigroups closed under finite intersections and for the Frobenius number, Houston J. Math. 34 (2008), 339-348.

[8] J. C. Rosales and M. B. Branco, Numerical semigroups that can be expressed as an intersection of symmetric numerical semigroups, J. Pure Appl. Algebra 171 (2002), 303-314.

[9] J. C. Rosales and P. A. García-SÁnchez, Numerical semigroups with embedding dimension three, Arch. Math. (Basel) $\mathbf{8 3}$ (2004), 488-496.

[10] J. C. Rosales and P. A. García-SÁnchez, Numerical Semigroups, Developments in Mathematics, Vol. 20, Springer, New York, 2009.

[11] H. S. Wilf, A circle-of-lights algorithm for the "money-changing problem", Amer. Math. Monthly 85 (1978), 562-565. 
84 M. A. Moreno-Frías and J. C. Rosales : Perfect numerical semigroups...

MARÍA ÁNGELES MORENO-FRÍAS

DEPARTMENT OF MATHEMATICS

FACULTY OF SCIENCES

UNIVERSITY OF CÁDIZ

E-11510, PUERTO REAL (CÁDIZ)

SPAIN

E-mail: mariangeles.moreno@uca.es

JOSÉ CARLOS ROSALES

DEPARTMENT OF ALGEBRA

FACULTY OF SCIENCES

UNIVERSITY OF GRANADA

AVENIDA DE LA FUENTE NUEVA S/N

C.P. 18071 , GRANADA (GRANADA)

SPAIN

E-mail: j.rosales@ugr.es

(Received July 19, 2019; revised December 33, 2019) 